\title{
A safer way of dry needling therapy for gastrocnemius muscles: ultrasound guidance
}

\author{
Fatih Bagcier ${ }^{1}$, Ozan Volkan Yurdakul ${ }^{2}$
}

${ }^{1}$ Department of Physical Medicine and Rehabilitation, Biruni University Faculty of Medicine, ${ }^{2}$ Department of Physical Medicine and Rehabilitation, Bezmialem University Faculty of Medicine, Istanbul, Turkey

\section{To the editor,}

Myofascial trigger points (MTrPs) in calf muscles may cause nocturnal cramps and radiating pain in the popliteal fossa and plantar fascia regions [1]. Various treatment methods have been applied, such as oral medication administration, invasive procedures, and exercise. Two of these invasive procedures, dry needling, and local anesthetic injection, have been demonstrated to have similar effects upon treatment [2].

Trigger points at the calf should be treated delicately because of the anatomic structure of the region. MTrPs of the proximal gastrocnemius (GC) muscle have critical anatomical contiguity due to the closerelationships with the popliteal fossa (fig 1a). The tibial nerve, popliteal artery, and vein pass through the two heads of the GC muscle (fig 1b). Also, the medial sural cutaneous nerve descends upon the medial border of the lateral head (LH) of the GC muscle, close to the border between the medial head $(\mathrm{MH})$ and the LH of the GC muscle (fig 1c). When performing invasive procedures to the MTrPs of this region, the practitioner should stay away from the midline and insert the needle with a lateral angle for $\mathrm{MH}$ or medial angle for LH (fig 1d). In addition, the practitioner should be careful regarding the common peroneal nerve when inserting the needle to the outer parts of the LH.

It is possible to perform safe injections by choosing the right needle size and proper positioning. For this rea-

Received 26.09.2020 Accepted 15.10.2020

Med Ultrason

2020, Vol. 22, No 4, 495-496, DOI: 10.11152/mu-2840, Corresponding author: Dr. Fatih Bagcier

Department of Physical Medicine and Rehabilitation, Biruni University

Faculty of Medicine, Istanbul

ORCID number: 0000-0002-6103-7873

Phone: +90 5442429042

Fax: +90 2122361014

E-mail: bagcier_42@hotmail.com

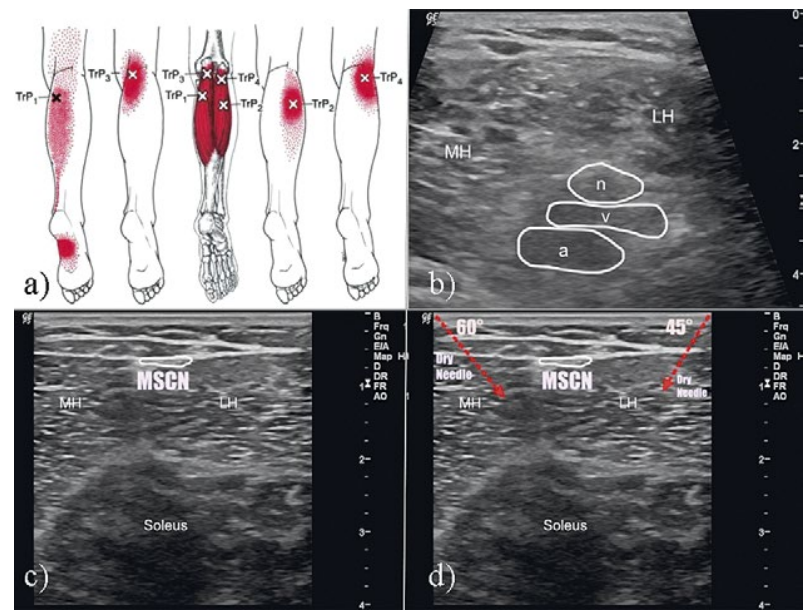

Fig 1. Locations for trigger points (a) in the gastrocnemius muscles. Locations of the tibial nerve, popliteal artery, vein (b), and medial sural cutaneous nerve (c), and safe angles for needle insertion (d). n: tibial nerve, a: popliteal artery, v: popliteal vein, MH: gastrocnemius medial head, LH: gastrocnemius lateral head, arrows: needle direction.

son, the penetration angle should be $45^{\circ}$ to the skin, and a $0.3 \mathrm{~mm} \times 30 \mathrm{~mm}$ sized sterile acupuncture needle should be used for LH's DN procedure. The procedure in the $\mathrm{MH}$ is safer, and a $0.3 \times 50 \mathrm{~mm}$ sterile acupuncture needle with $60^{\circ}$ of penetration angle is usually enough. For the GC muscle, the proper position of the patient is lying prone with knees in slight flexion.

The use of the US in guiding the procedures may minimize possible complications [3], for example, the lesion of the neurovascular structures.

In this case, the dry needling of this anatomical region under US guidance was a safe procedure. The superiority of US-guided methods to blinded injections may be a matter of debate, and randomized controlled trials are required to clarify this point. We should keep in mind that myofascial pain syndrome and MTrPs are considered as underdiagnosed diseases. We believe that the utilization 
of US may offer different procedures regarding the treatment of this condition [4].

\section{References}

1. Kim DH, Yoon DM, Yoon KB. The effects of myofascial trigger point injections on nocturnal calf cramps. J Am Board Fam Med 2015;28:21-27.

2. Küçük Eroglu P, Yilmaz Ö, Bodur H, Ateş C. A Comparison of the Efficacy of Dry Needling, Lidocaine Injection, and
Oral Flurbiprofen Treatments in Patients with Myofascial PainSyndrome: A Double-Blind (For Injection, Groups Only), Randomized Clinical Trial. Turk J Rheumatol 2013;28:38-46.

3. Ricci V, Özçakar L. Ultrasound imaging of the upper trapezius muscle for safer myofascial trigger point injections: a case report. Phys Sportsmed 2019;47:247-248.

4. Abdulsalam AJ, Mezian K, Ricci V, et al. Interdigital Approach to Trigger Finger Injection Using Ultrasound Guidance. Pain Med 2019;20:2607-2610.

\title{
Ultrasound examination is doubly effective for hoarseness: imaging for the vocal cords and the recurrent laryngeal nerve
}

\author{
Ke-Vin Chang ${ }^{1}$, Wei-Ting Wu ${ }^{1}$, Levent Özçakar ${ }^{2}$
}

${ }^{1}$ Department of Physical Medicine and Rehabilitation, National Taiwan University Hospital, Bei-Hu Branch, Taipei, Taiwan, ${ }^{2}$ Department of Physical and Rehabilitation Medicine, Hacettepe University Medical School, Ankara, Turkey

\section{To the Editor,}

A 45-year-old male had been diagnosed with esophageal squamous cell carcinoma two years ago when esophagectomy and lymph node dissection had also been performed. Since then, he had been under regular followup with remission. Having suffered gradual hoarseness for the last three months, he was referred for an ultrasound (US) examination. As his chief complaint was hoarseness, the transducer was placed over the thyroid cartilage to evaluate vocal cord movements. As reduced motion was observed on the right side (fig 1A, Video 1, on the journal site), vocal cord palsy was suspected. Accordingly, the transducer was relocated towards the right lateral neck to scan the recurrent laryngeal nerve (RLN) - seen as a hypoechoic fascicle interposed between the posterior border of the thyroid gland and the lateral edge of the tracheal ring (fig 1B). Moving the transducer more caudally, a hypoechoic circular mass was detected at the location of RLN (fig 1C). Redirecting the transducer in the coronal oblique plane, the RLN was seen in continuation with - and enveloped by - the tumor (fig 1D). The following positron

Received 07.07.2020 Accepted 15.09.2020

Med Ultrason

2020, Vol. 22, No 4, 496-497, DOI: 10.11152/mu-2713,

Corresponding author: Ke-Vin Chang, MD, PhD

Department of Physical Medicine and Rehabilitation, National Taiwan University Hospital, Bei-Hu Branch and National Taiwan University College of Medicine, Taipei, Taiwan E-mail: kvchang011@gmail.com

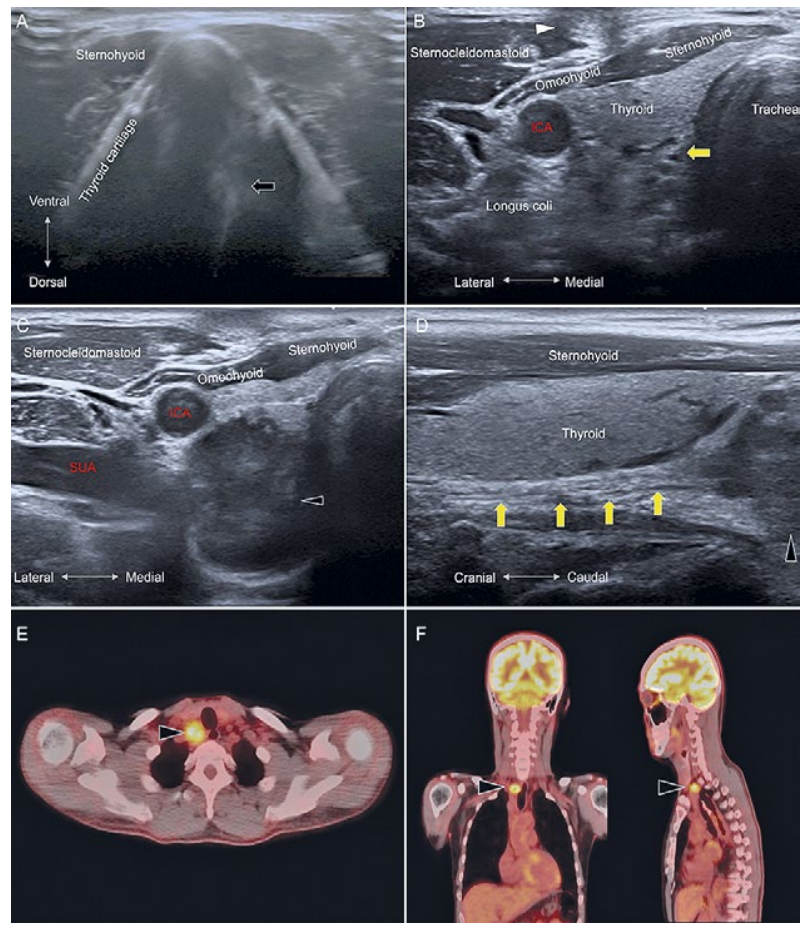

Fig 1. Ultrasound imaging of normal vocal cord vibrations (black arrow) at the non-paralytic site (A). Short-axis tracking of the right recurrent laryngeal nerve (yellow arrowhead) cranial (B) and caudal (C) to the metastatic tumor (black arrowhead). Long-axis view of the nerve (D). Positron emission tomography images show the metastatic tumor in the horizontal (E), and coronal/sagittal planes (F). White arrowhead: operation scar; ICA, internal carotid artery; SUA, subclavian artery. 
emission tomography scan confirmed a hypermetabolic mass over the right paratracheal region (fig 1E, 1F). Neck lymph node biopsy revealed metastatic squamous cell carcinoma and radiotherapy was planned accordingly.

US serves as a convenient tool for the evaluation of vocal cord movements [1]. Vocal cord palsy is primarily derived from disorders of the RLN which innervates all laryngeal muscles but the cricothyroid muscle. After branching from the vagus nerve, the right and left RLN loop around the subclavian artery and the aortic arch, respectively [2]. To visualize the right RLN at the neck level, the transducer should be positioned over the right paratracheal area [3]. The nerve can be recognized medial to the longus coli muscle, posterior to the lateral lobe of the thyroid gland, next to the tracheal ring. Except for laterality, location of the left RLN is similar to the right one but with the esophagus situated underneath it. This present case highlights the utility of US with regard to the evaluation of vocal cord dysfunction as well as relevant/ accompanying pathologies involving the RLN.
Acknowledgment: The current research project was supported by 1) National Taiwan University Hospital, Bei-Hu Branch; 2) Ministry of Science and Technology (MOST 106-2314-B-002-180-MY3 and 109-2314-B002-114-MY3); and 3)Taiwan Society of Ultrasound in Medicine.

\section{References}

1. Finnoff JT, Orbelo DM, Ekbom DC. Identification of Paradoxical Vocal Fold Movement with Diagnostic Ultrasound: Confirmation with Video Laryngoscopy. PM R 2020;12:425-427.

2. Solbiati L, De Pra L, Ierace T, Bellotti E, Derchi LE. Highresolution sonography of the recurrent laryngeal nerve: anatomic and pathologic considerations. AJR Am J Roentgenol 1985; 145:989-993.

3. Chang KV, Lin CP, Hung CY, Ozcakar L, Wang TG, Chen WS. Sonographic Nerve Tracking in the Cervical Region: A Pictorial Essay and Video Demonstration. Am J Phys Med Rehabil 2016;95:862-870.

\title{
Ultrasound as a complementary diagnostic method for Dupuytren's Contracture
}

\author{
Mustafa Turgut Yildizgoren
}

Fizikon Medical Center, Department of Physical Medicine and Rehabilitation, Konya, Turkey

\section{To the Editor,}

Dupuytren's contracture (DC) is a benign fibrotic cord or nodule on the palmar fascia of the hand resulting in digital flexion contractures. Clinical findings of palmar fibromatosis include palpable firm superficial nodules or cords, possible tenderness, involving the palmar aspect of the hand, with debilitating flexion contractures of the fingers [1]. DC is a clinically distinct entity that is rarely misdiagnosed once the syndrome is well estab-

Received 26.07.2020 Accepted 25.09.2020

Med Ultrason

2020, Vol. 22, No 4, 497-498, DOI: 10.11152/mu-2657,

Corresponding author: Mustafa Turgut Yildizgoren, MD

Fizikon Medical Center, Department of

Physical Medicine and Rehabilitation

Konya, Turkey

Phone/Fax: 903323536900

E-mail: ftr.mustafaturgut@hotmail.com lished. Magnetic resonance imaging (MRI) or ultrasound may be helpful in considering the differential diagnosis including tenosynovitis, trigger finger, ganglion, dermoid cyst, soft-tissue masses or for imaging of vascular complications. Presentation in patients younger than 40 years is unusual, in which case an underlying condition, such as sarcoma, needs to be excluded with MRI [2]. Although the current gold standard for the diagnosis of DC is the physical examination of the hand, this only provides 2-dimensional information about the disease. Ultrasound examination provides both static and dynamic assessment of the tendon and adjacent soft-tissue [3]. Ultrasound is useful for determining whether a mass is cystic or solid, assesing its degree of vacularity, the measuring of disease activity, providing real-time guidance during therapeutic procedures, and the follow-up of treatment of patients. The nodules' adherence to the tendons can be demonstrated with dynamic studies [1]. 
A 62-year-old female presented with nodular thickening of the fourth finger on the right hand. She stated that the problem had developed slowly and worsened over several months. The anamnesis and physical examination were suggestive of DC. The examination revealed a wellpalpable nodular band of cohesive tissue on the metacarpal of the right-hand fourth finger. Range of motion was normal. Ultrasound examination was also performed, which confirmed the presence of palmar fibromatosis at the level of the distal metacarpal and above the flexor tendons of the fourth finger (fig 1). On dynamic ultrasound examination, the tendons below the palmar fibromatosis were observed to move smoothly (Video 1, on the journal site). The patient was evaluated as early phase DC and physical therapy was initiated, including therapeutic ultrasound, splinting, massage, and joint stretching. The findings were evaluated by clinical examination and the patient reported softening and reduced thickening of nodules at the end of treatment.

Ultrasound has been widely used to viewing the palmar fascia to confirm DC and for ultrasound-guided treatment. Ultrasound guidance is also required to avoid the complications associated with injections into tendons, neurovascular structures and collagen-containing structures within the hand.

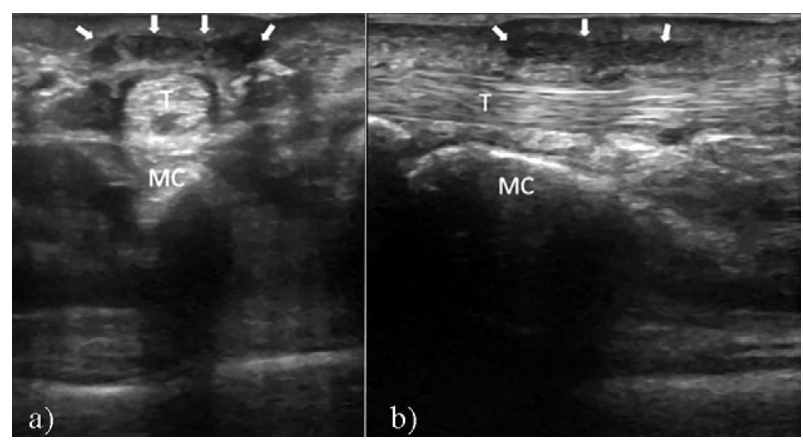

Fig 1. Transverse (a) and longitudinal (b) ultrasound images showing hypoechoic nodular thickening (arrows) at the level of the distal metacarpal (MC) and above the flexor tendons (T) of the fourth digit.

\section{References}

1. Morris G, Jacobson JA, Kalume Brigido M, Gaetke-Udager K, Yablon CM, Dong Q. Ultrasound Features of Palmar Fibromatosis or Dupuytren Contracture. J Ultrasound Med 2019;38:387-392.

2. Waldman SD. Dupuytren's contracture. In: Pain review. Philadelphia: Saunders; 2009:277-278.

3. Molenkamp S, van Straalen RJM, Werker PMN, Broekstra DC. Imaging for Dupuytren disease: a systematic review of the literature. BMC Musculoskelet Disord 2019;20:224.

\title{
A modified ultrasound-guided supraclavicular brachial plexus block for the operative fixation of the humerus head and shaft fractures
}

\author{
Adela Hilda Onutu ${ }^{1}$, Elena Retegan ${ }^{1}$, Catalin Constantinescu ${ }^{2}$, Gabriella Iohom ${ }^{3}$
}

\begin{abstract}
${ }^{1}$ Anaesthesia and Intensive Care Compartment, Orthopedic Department Emergency County Hospital Cluj-Napoca, Romania, ${ }^{2}$ Anaesthesiology and Intensive Care Department, "Iuliu Hatieganu" University of Medicine and Pharmacy, Cluj-Napoca, Romania, ${ }^{3}$ Anaesthesiology and Intensive Care Medicine Department, Cork University Hospital, Cork, Ireland
\end{abstract}

\section{To the Editor,}

Recently the supraclavicular approach of the upper trunk block at the point of its division was described [1].

Received 21.08.2020 Accepted 29.09.2020

Med Ultrason

2020, Vol. 22, No 4, 498-499, DOI: 10.11152/mu-2790,

Corresponding author: Adela Hilda Onutu, MD, PhD

43-47 General Traian Mosoiu street,

Cluj-Napoca, Romania

E-mail: adela_hilda@yahoo.com
Previous studies described low interscalene approaches for elbow and distal forearm surgery but made no reference to shoulder surgery [2].

Herein, we present the anaesthetic management of a 61-year man admitted with polytrauma (bilateral temporomandibular luxation, facial wounds, multiple rib fractures and comminuted head and shaft fracture of his left humerus). Given his facial trauma and comorbidities, we chose a regional anaesthesia technique for surgery, to avoid face mask ventilation and tracheal intubation. With 
standard monitoring and intravenous access, the patient was positioned supine with $30^{\circ}$ head up. Midazolam 1 $\mathrm{mg}$ was administered intravenously before the procedure. After skin disinfection and probe preparation, we placed the linear transducer (12.6 MHz, UGEO PT60A, Samsung, South Korea) above and parallel to the left clavicle to identify the subclavian artery and the cluster of nerve structures lateral to it. We rotated then the probe anti-clockwise approximately $45^{\circ}$, obtaining a good image of all three trunks of the brachial plexus (BP): superior, middle, and inferior (fig 1). A 22G, $50 \mathrm{~mm}$ insulated needle (Uniplex Nanoline, Pajunk, Germany) was advanced in-plane from lateral to medial towards each trunk and $30 \mathrm{ml}$ ropivacaine $0.5 \%$ was deposited in increments of $3 \mathrm{ml}$ following repeated negative aspiration. In addition, $5 \mathrm{ml}$ of ropivacaine $0.5 \%$ was deposited deep to the sternocleidomastoid muscle (at C6 level) to block the superficial cervical plexus. After 20 minutes the patient had a complete sensory and motor block in all innervation areas of the BP. Surgery proceeded uneventfully for 90 minutes and the first postoperative analgesic requirement was recorded 13 hours postoperatively.

Placing the transducer as described allowed a more superficial approach $(1-1.5 \mathrm{~cm})$ of BP block, with better visualization of trunks, and diminished the risk of accidental pleural puncture.

The main advantage of this technique is, however, a more superficial location of the middle and lower trunks/ divisions, allowing in some subjects, a complete block of the upper limb. A lower incidence of phrenic nerve palsy may be a potential advantage, but this requires further investigation.

The main disadvantage lies with the prevailing large anatomical variation among individuals which makes the lower trunk/posterior divisions difficult to block in some patients. However, the upper trunk block on its own as

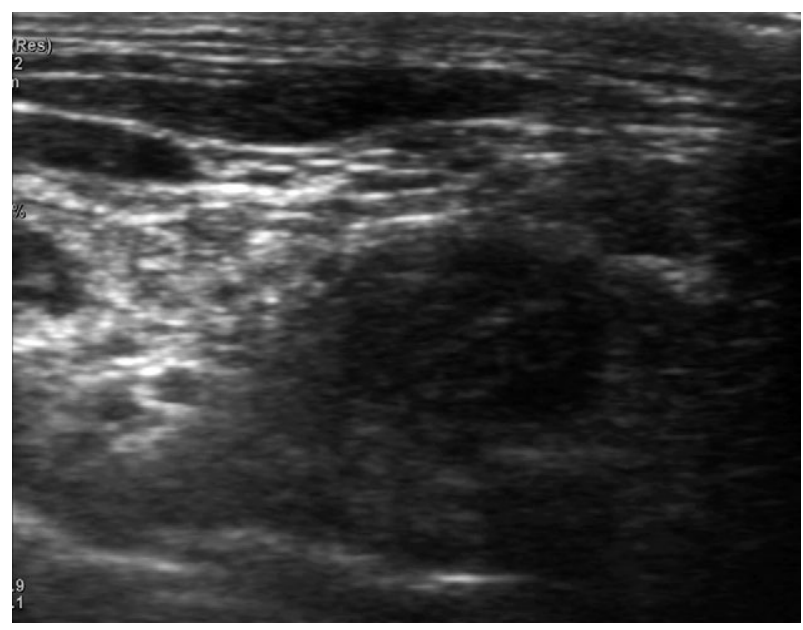

Fig 1. The needle targeting the superior trunk

part of a multimodal analgesia regimen provides sufficient analgesia for shoulder surgery [3].

This modification of the supraclavicular approach to the BP block may prove a useful addition to the regional anaesthesia armamentarium.

\section{References}

1. Aguirre O, Tobos L, Reina MA, Sala-Blanch X. Upper trunk block: description of a supraclavicular approach of upper trunk at the points of its division. Br J Anaesth 2016;117:823-824.

2. Gadsden JC, Tsai T, Iwata T, Somasundaram L, Robards C, Hadzic A. Low interscalene block provides reliable anesthesia for surgery at or about the elbow. J Clin Anesth 2009;21:98-102.

3. Kang R, Jeong JS, Chin KJ, et al. Superior trunk block provides noninferior analgesia compared with interscalene brachial plexus block in arthroscopic shoulder surgery. Anesthesiology 2019;131:1316-1326. 


\title{
Developing and using a smartphone-app to support the teaching of technical basics for 3D/4D obstetrical ultrasound applications in medical education
}

\author{
Christian Kollmann', Lukas Santner ${ }^{1}$, Jo Yong-Ha²
}

${ }^{1}$ Center for Medical Physics \& Biomed. Engineering, Medical University of Vienna, Austria, ${ }^{2}$ College of Medicine, University of Ulsan, Seoul, South Korea

\section{To the Editor,}

Medical students are becoming familiar with ultrasound imaging early in the regular curriculum during their study. Mainly medical aspects are presented while only short lectures on the basic technological background are given in the sessions or this needs to be worked out by the students themselves using teaching books, e-books or internet. This self-instruction is mostly not supervised nor are the information sources quality-controlled. The outcome is that students are often unprepared for working with ultrasound scanners in skills-labs and require basic methodical introductions again.

Therefore, we have developed a concept to reinforce the basic skills of students to know and understand the technology behind a scanner [1]. Different electronical resources have been developed and are offered freely to the students at our university within a special repository. Several years ago, our University implemented a medical eEducation environment (m3e) which collected and offered validated resources to the students for different medical fields. The app is described below and is made available to everyone [2].

The latest resource has been an Android-based smartphone-app to instil the basic technical principles and medical applications of 3D/4D-ultrasound in obstetrics. This app has been compiled with the open source software Android Studio (Vers.3.3.1, [3]) and runs on mobile phones or tablets using the Android version 4.4 (Kitkat)

Received 11.08.2020 Accepted 28.09.2020

Med Ultrason

2020, Vol. 22, No 4, 500-501, DOI: 10.11152/mu-2772,

Corresponding author: Christian Kollmann

Center for Medical Physics \&

Biomed. Engineering,

Medical University of Vienna, Austria

e-mail: christian.kollmann@meduniwien.ac.at

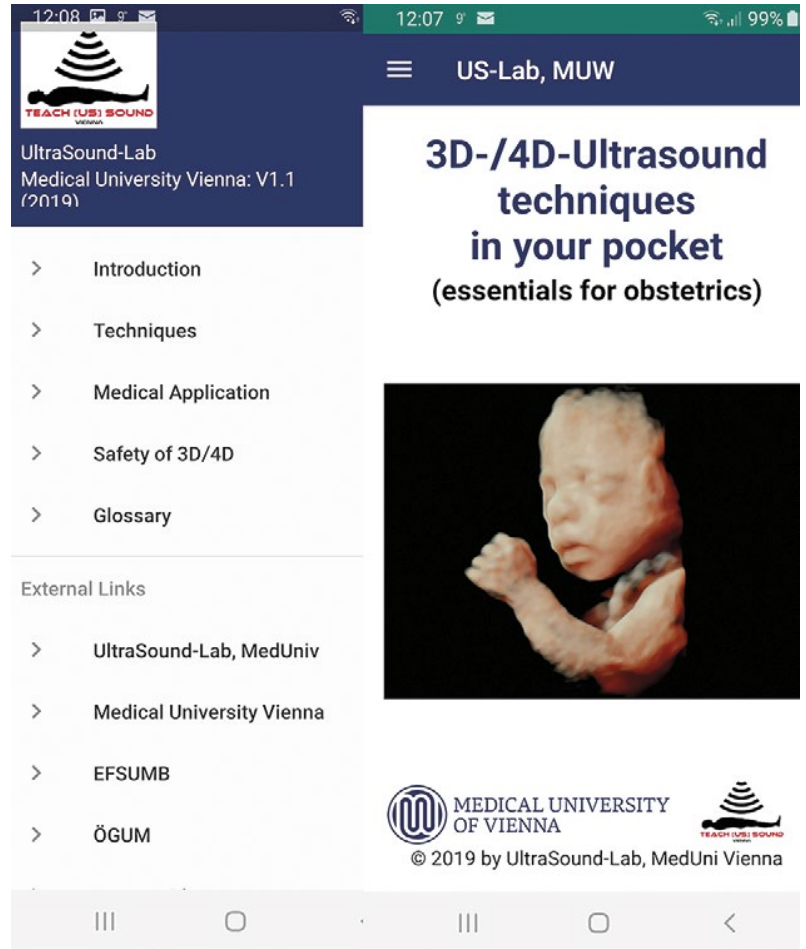

Fig 1. Collage of the home screen and content menu of the smartphone app for 3D/4D obstetrical ultrasound

and later versions [4]. It contains different pages offering basic essential facts regarding techniques, safe use and medical applications in obstetrical screening by using videos and graphics. A glossary and external links to the topic are included, too (fig 1).

With this app the students have all the relevant information in their "pocket". A fast feedback among our skills-lab students (12 from 42, 28.6\% return rate) using a standardised questionaire showed that the highest rating was given to our balanced learning content and the selection of relevant videos and graphics on the topic. All 
students recommended this app to their fellow students, therefore, we decided to make it public to all medical students using the share button within the app or downloaded from our university's m3e-website [3].

Acknowledgement: MD-candidate J. Y-H. has been supported by the Austrian Medical Students' Association (AMSA) and exchanged her special knowledge of medical practice and this application with us, while BSccandidate S.L. set up the programming in a short time and with an excellent outcome.

\section{References}

1. Kollmann C. Teaching Technical Basics in Ultrasound for Medical Students - Outline of the Viennese 'Teach Us Sound' Concept. Praxis 2018;107:1273-1278.

2. Medical University Vienna. m3e - Medical eEducation Environment.Available from:https://m3e.meduniwien.ac.at $/ \mathrm{m} 3 \mathrm{e} /$

3. Android Studio. Google Mountain View, USA. Available from:https://developer.android.com/studio

4. Medical University Vienna. 3D/4D-ultrasound techniques in your pocket. e-resource database. Available from: https:// m3e.meduniwien.ac.at/download/US_Lab/ 